\title{
Bacteriological Assessment of Hospital Air and Selected Surfaces in Three Referral Hospitals in Makurdi Middle Belt Nigeria
}

\author{
Emmanuel Olumuyiwa Onifade ${ }^{1^{*}}$, Innocent Okonkwo Ogbonna ${ }^{1}$, Joseph Ikwebe ${ }^{2}$, Stephen Olaide Aremu ${ }^{1,3}$, \\ Tosin Adebola Ode ${ }^{4}$, Itodo Samuel Olusegun ${ }^{5}$, Oluwole Opeyemi Owoyemi ${ }^{6}$
}

\author{
${ }^{1}$ Federal University of Agriculture, Makurdi, Benue State, NIGERIA \\ ${ }^{2}$ Federal University Wukari, Taraba State, NIGERIA \\ ${ }^{3}$ Siberian State Medical University, Tomsk Region, RUSSIA \\ ${ }^{4}$ Salem University, Lokoja, kogi State, NIGERIA \\ ${ }^{5}$ Benue State University, Makurdi, NIGERIA \\ ${ }^{6}$ Achievers University, Owo, Ondo State, NIGERIA \\ *Corresponding Author: olumuyiwa.onifade@yahoo.com
}

Citation: Onifade, E. O., Ogbonna, I. O., Ikwebe, J., Aremu, S. O., Ode, T. A., Itodo, S. O. and Owoyemi, O. O. (2020). Bacteriological Assessment of Hospital Air and Selected Surfaces in Three Referral Hospitals in Makurdi Middle Belt Nigeria. European Journal of Environment and Public Health, 4(2), em0056. https://doi.org/10.29333/ejeph/8454

\section{ARTICLE INFO}

Received: 24 Apr. 2020

Accepted: 22 Jul. 2020

\begin{abstract}
Hospital environment needs more attention because of the influx of people into the environment for medical checkup and other services. This study unveils the incidence of bacteria isolated from air and selected surfaces in three referral hospitals (City hospital, Federal Medical Centre, Wadata and Saint Theresa Hospital) in Makurdi Middle Belt Nigeria. Fifty (50) bacteria were isolated; hospital air (26\%), bed rails (16\%), door knobs (2\%), floor (4\%), nurse table top (10\%), operation table (4\%), sink (18\%), stretchers (2\%) and toilet seat (12\%). In City Hospital, the bacteria isolated include Escherichia coli (6\%), Pseudomonas aeruginosa (2\%), Staphylococcus aureus and Staphylococcus spp (2\%), from Federal Medical Centre, bacteria isolated were Klebsiella spp (10\%), Staphylococcus aureus (4\%), Staphylococcus spp (8\%), Pseudomonas aeruginosa (12\%) and E. coli (6\%). In STH, bacteria isolated include E. coli (8\%), Pseudomonas aeruginosa (4\%), Staphylococcus spp (8\%) and Staphylococcus aureus (14\%). The invitro antibiotics susceptibility pattern shows that Pseudomonas aeruginosa showed highest resistant to the antibiotics while Klebsiella spp was susceptible to majority of the antibiotics but resistant to cloxacillin and erythromycin. The study reveals Klebsiella spp, Escherichia coli, Pseudomonas aeruginosa, S. aureus and other Staphylococcus spp as bacteria commonly associated with hospital environment. This study affirms the presence of resistant bacteria strains and highlighted world-wide problem of hospital borne infections as it concerns the study area and population. This report will create awareness and be a good guide to health care workers, patients and the public about the likeliness of contracting nosocomial infection and how to treat such infection. Major recommendations offered suggests that, healthcare workers should be more careful in carrying out their duty to avoid chance of being infected in the course of their work. Also, the in-vitro antibiotics susceptibility testing on the bacterial pathogens in the study will assist the clinicians in making improvement on the management of nosocomial infections.
\end{abstract}

Keywords: surface swabs, hospital environments, pathogens, in-vitro, antibiotics

\section{INTRODUCTION}

The microbes operate in different ecosystem because in their world they are exceedingly diverse in their appearance, metabolism, physiology, and genetics. The incredible biodiversity bacteria range from extreme environment in water even unto the atmosphere in air. This variety of species is as well present in a particular environment like hospitals air and some surfaces in hospitals (Prescott, Harley, and Klein, 2008).
Hospital environments are places that need more attention because of the influx of people coming for medical checkup and other services in the environment. Patients and hospital staff get in contact with this environment every day and some of these patients may have little or no knowledge about the bacteriology of the hospital air and various surfaces that are potential source of infections. Coronavirus disease (COVID19) pandemic has provided a lot of information about air borne infection, there is need to also shed light on bacteria that are capable of causing infection from hospital premises also known as nosocomial infection. These infections often manifested within forty-eight hours after a patient is being 
admitted or thirty days after being discharge from the hospital (Anusree et al., 2015; WHO, 2002). Therefore, bacteria from air can cause infection on patients with compromised immunity, especially when the patients come close a very close range (that is, neglecting physical distancing) to another individual who is a carrier of bacteria that are potential hospital acquired pathogens. So, exchange of gas due to respiration during conversation or exchange of pleasantry will pave way to inhale potential pathogen breathe out by another person at closed range.

Therefore, there is likeliness of contracting nosocomial infection by patient and, or healthcare staff from the hospital environment which is a major route of nosocomial bacterial pathogens (Tambekar, Gulhane, and Bhokare, 2007). At Kenyatta National Hospital, Kenya, P. aeruginosa, Klebsiella, Citrobacter, S. aureus, S. pneumoniae, Acinetobacter and E. coli were isolated from tracheal aspirate, urine, blood and pus swabs from the intensive care unit (Peter et al., 2017). Some researchers have highlighted a host of nosocomial bacteria which include Streptococcus spp, Acinetobacter spp, Enterococci, Pseudomonas aeruginosa, coagulase-negative Staphylococcus, Staphylococcus aureus, Bacillus cereus, Legionella, Proteus mirablis, Klebsiella pneumonia, E. coli, and Serratia marcescens (Bereket et al., 2012; Drach, 2005; Hassan, Aftab, and Riffat, 2015; Stamm, 2001). Even though, efforts are made in Nigeria through awareness of the general public on airborne infection of COVID -19 which is a viral disease, but there is little awareness on airborne infection caused by bacteria. Hence, the microbiological quality assessment of indoor air is one of the most vital investigations to determine the microbial indoor air pollution. The information on the indoor microbial concentrations of airborne bacteria is necessary both to estimate the health hazard and to create standards for indoor air-quality control (Arzu et al., 2019).

Fu and Wang (2016) reported nosocomial infection control in health care settings with respect to protection against emerging infectious diseases; the report revealed that proper control measure should be taken to ensure that nosocomial infections do not occur in designated healthcare settings that accommodate suspected cases suffering from emerging infectious diseases and a comprehensive and detailed evaluation of nosocomial infection control should be conducted in each designated healthcare setting.

Moreover, hospital environment and its air are inseparable from health care worker and patients, bacteriological assessments of the environment such as indoor air quality cannot be underestimated when it comes to human health. The bacteria that live in troposphere, stratosphere and even ionosphere are capable of causing indoor air pollution (Awosika, Olajubu, and Amusa, 2012; Tambekar, Gulhane, and Bhokare, 2007).

Favorable temperature and poor air ventilation of indoors coupled with poorly designed structure gives an ample opportunity for microbial growth. It has been observed that Bacillus, Clostridium and Staphylococci are the most common bacteria isolated from indoor surfaces (Hayleeyesus and Manaye, 2014). Tambekar, Gulhane, and Bhokare (2007) report showed that bacteria can survive in both indoors and outdoors and the maternity and children hospitals had the highest bacterial isolates. It is easier to avoid infection transmitted from formites than that of airborne which is often difficult.

However, lithosphere is conducive for a lot of mixed microbes which may become airborne and exposure to this may cause an epidemic infection of a particular environment because contaminated air is significant in transmission of communicable airborne diseases (Klevens et al., 2007; Tambekar, Gulhane, and Bhokare, 2007). It is obvious that hospital acquired infection is on the increase and the rate of antibiotic resistance of bacterial pathogens associated with these infections is alarming in the public health (Wondemagegn et al., 2012).

Consequently, to avoid the risk of infection by nosocomial bacteria, negative-pressure quarantine ICUs and wards should be built in accordance with the demand in the area. Regulations, standards, procedures, and operational instructions on protection against infections from emerging respiratory, gastrointestinal, body fluid, and insect-borne infectious diseases should be established. Periodical regular trainings for the knowledge of prevention and control emerging or unknown infectious diseases, and emergency exercises regarding nosocomial infection events among medics can also help to strengthen the infection control system (Fu and Wang, 2016).

According to previous investigations, the individual and nations economy do suffer loss due to the menace of this infections thereby causing financial burden, disability of patients, morbidity and eventual death also known as mortality of the hospitalized victims. The victim may be infected via surgical wound, blood stream, respiratory, urinary tract infections (Endalafer, Gebre-Selassie, and Kotisso, 2010;; Wondemagegn et al., 2012). Wondemagegn et al. (2012) isolated $26.2 \% S$. aureus as the leading bacterial isolates followed by E. coli and coagulase negative Staphylococcus spp. which consist of $21.4 \%$ each. Wondemagegn et al. (2012) further revealed that almost all the bacteria in the study were resistant to at least two of the antibiotics employed.

In general, bacteriological study of hospital environment has revealed the incidence of some bacteria associated with the environment. This research is aimed at bacteriological assessment of hospital air and selected surfaces in three referral hospitals in Makurdi, middle belt, Nigeria.

\section{MATERIALS AND METHODS}

\section{The Study Area and Population}

The study areas of the research are three referral hospitals which include: City Hospital $(\mathrm{CH})$, Federal Medical Centre Wadata (FMCW) and Saint Theresa Hospital (STH) in Makurdi, Nigeria. One hundred and forty-three (143) samples which include 104 surface swabs and 39 samples from air were collected from the referral hospitals for laboratory analysis within a period of five months.

\section{Ethical Clearance}

The consent of the administrative authorities of Federal Medical Centre Wadata, Saint Theresa Hospital and City 
Hospital were obtained before commencement of the research in the hospitals.

\section{Collection of Samples}

Air samples were taken by use of plate exposure methods while the surface swabs of the hospitals environments were collected aseptically by the use of sterile swabs sticks by trained personnel and researchers in the morning before commencement of work and transported immediately to the Microbiological Laboratory of Federal University of Agriculture, Makurdi. The parts of the hospital environment where patients, hospital staff and even visitors do have contact with almost every time were used as sample site due to likelihood of being infected. Thus, the analyses of samples collected are as follows:

(i) 40 samples were taken from City Hospital (C): Thirty (30) samples from selected surfaces and 10 from hospital air. The samples collected from the surfaces include nurse table tops (4), bed rails (3), door knobs (4), from stretchers (3), sinks (4), operation table (4), hospital floor (4), and toilet seats (4), while 10 samples were collected from the hospital air.

(ii) 47 samples were collected from Saint Theresa Hospital (S): 32 surface swabs which include nurse table tops (4), bed rails (4), door knobs (4), stretchers (4), sinks (4), operation table (4), hospital floor (4), toilet seats (4) and 15 samples were collected from the air of maternity ward (labour room), female medical ward, male medical ward and pediatrics ward.

(iii) A total of 56 were collected from Federal Medical Centre Wadata $(F): 42$ samples were collected from the surfaces and 14 samples from the hospital air. The samples collected from the surface swabs include nurse table tops (6), bed rails (6), door knobs (6), stretchers (6), sinks (6), hospital floor (6) and toilet seats (6) and air samples (14) were obtained from Federal Medical Centre.

\section{Materials Used}

The materials used for the study include: Bacteriological Media (Nutrient agar, Chocolate agar, Blood agar and MacConkey agar), sterile swab stick, glass wares (Petri dishes, round bottom flask, graduated cylinder, conical flask glass, slides) and antibiotic sensitivity disc. Other apparatus, reagents and equipment include incubator, autoclave, forceps, inoculating loop, Bunsen burner, aluminum foil, cotton wool, weighing balance, work bench, peptone water, distil water, safranin, alcohol and Lugol's iodine (Cheesbrough, 2006).

\section{Procedure for Sterilization}

All glass wares were sterilized as described by Cheesbrough (2006).

\section{Sampling Design and Techniques}

Air samples were taken by exposure of prepared media in plates (set plates) into air for 20 minutes while surface swabs were collected using sterile swab sticks. The selected surfaces analyzed include hospital floors, door knobs, nurse table tops, bedrails, stretchers, operation tables, toilet seats and sinks for the purposes of isolating microorganisms from these sources and transported with sealed sterile polythene bag to the laboratory for analysis within thirty minutes. These samples were inoculated into nutrient agar plates and blood agar under aseptic condition as described by Cheesbrough (2006).

\section{Method of Isolation}

\section{Cultural method}

The swabs collected from the selected surfaces by the researchers and other trained medical laboratory scientist were streaked on the bacteriological media with the use sterile swab sticks to make an inoculum on the plates. These swabs samples were taken before cleaning of the hospitals while, the bacteria from air samples were isolated by plate exposure method before incubation and later streaked with the use of a sterile wire loop to make axenic culture (Prescott, Harley, and Klein, 2008).

\section{Isolation and characterization of isolates}

The organisms cultured from the air and the swabs were directly inoculated on nutrient agar by the use of sterile inoculating loop and later sub-cultured on blood agar, chocolate and MacConkey agar near bunsen burner. The inoculated media were incubated at $35^{\circ} \mathrm{C}$ for 24 hours and then examined for bacterial growth. The organisms isolated were identified by cultural characteristics, microscopy (Gram stain) and biochemical tests which include catalase, coagulase test, indole test, citrate test, oxidase test and carbohydrates fermentation (Prescott, Harley, and Klein, 2008).

\section{In-vitro Determination of Antibiotic Susceptibility}

Antibiotic susceptibility of the bacterial isolates was performed by disk diffusion method according to Clinical Laboratory Standards Institute guidelines. The bacteria were sub-cultured to prepare the inoculum with the sterile inoculating loop. Then, one isolated colony was transferred into $10 \mathrm{ml}$ of sterile distilled water prepared in the test tube and mixed properly. The liquid now serve as source of inoculum containing $10^{6} \mathrm{CFU} / \mathrm{ml}$ of bacterial suspension. A sterile swab stick was properly dipped into mixed inoculum and excess fluid was removed before rotating the swab on the surface of the prepared Mueller-Hinton agar plates. This spread was done four different planes by rotating the planes $180^{\circ}$ each time. This was to ensure that every part of the plate was inoculated. Forceps was flammed until red hot and cooled and then used to pick the antibiotics disc and it was also used to place the discs on the inoculated plates. The discs were pressed down on the agar so as to be in complete contact with agar. The plates were incubated at $37^{\circ} \mathrm{C}$ for 18 hours. The antibiotics multidisc employed contained Ofloxacin (OFL) $5 \mu \mathrm{g}$, Ceftriazone (CTR)

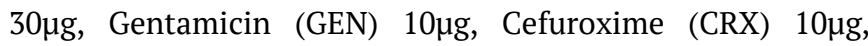

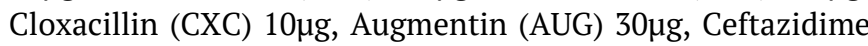

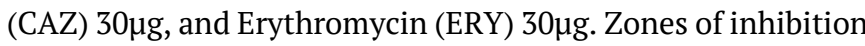
were used to determine the level of susceptibility of the isolates to these test antibiotics (CLSI, 2012).

\section{Statistical Analysis}

Statistical Package for the Social Sciences (SPSS) software version 23 was used for data analysis through the use of descriptive statistics which include frequency tables, 


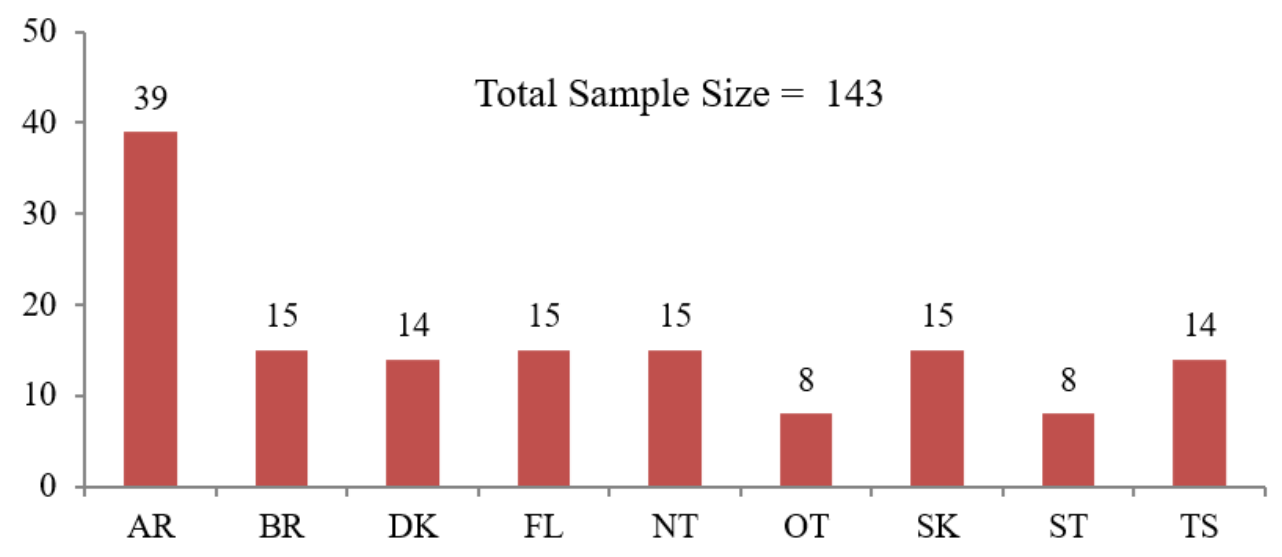

Figure 1. Distribution of Bacteriological Samples Key: AR- Air Sample, BR- Bed Rails, DK- Door knobs, ST- Stretchers, SK-Sink, TS - Toilet seat, OT - Operation table, FL - Floor, TS - Toilet Seat, NT - Nurse Table Top

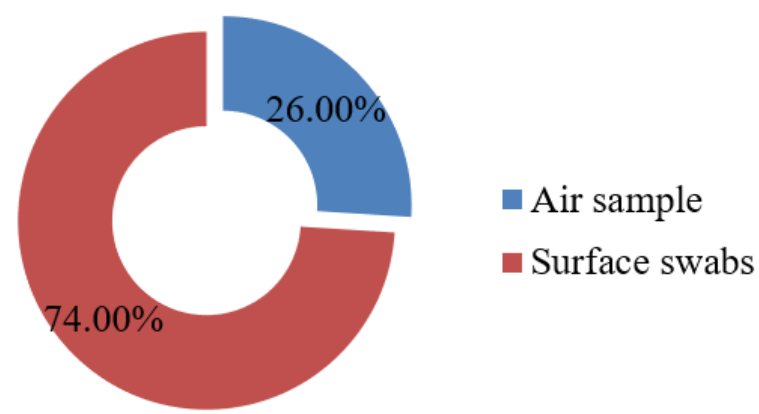

Figure 2. Occurrence of the Bacterial Isolates in Relation to Air Sample and Surface Swab

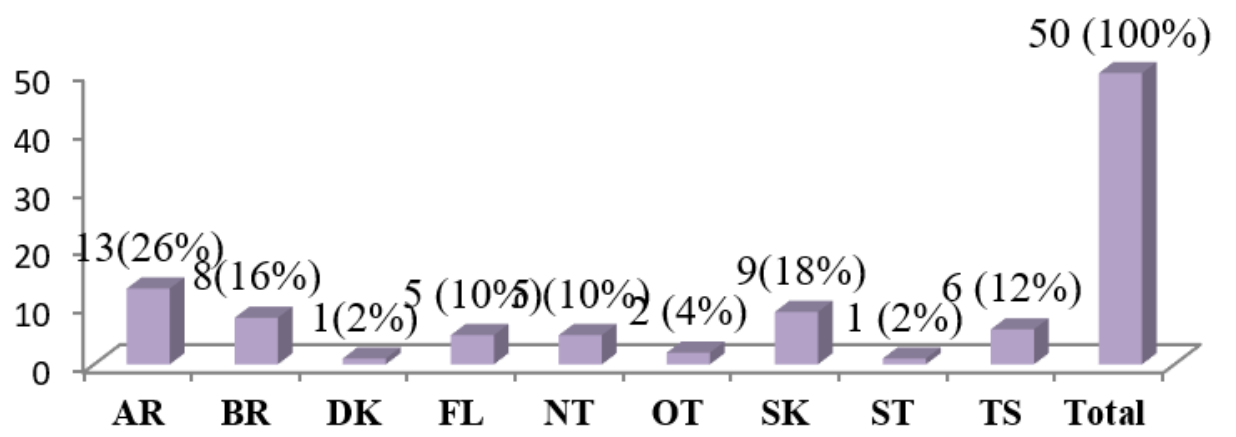

Figure 3. Incidence of Bacterial Isolates from Hospital Air Sample and Selected Surfaces Key: AR- Air Sample, BR- Bed Rails, DK- Door knobs, ST- Stretchers, SK-Sink, TS - Toilet seat, OT - Operation table, FL - Floor, TS - Toilet Seat, NT - Nurse Table Top

percentage, pie chart and bar chart as described by Pallant (2007).

\section{RESULTS AND DISCUSSION}

The bacteriological assessment of the three referral hospitals is presented below. Figure 1 depicts the distribution of bacteriological samples while Figure 2 represents the occurrence of the bacterial isolates in relation to air sample and surface swab. Figure 3 reveals the incidence of bacteria isolated from the air sample and selected surfaces of the referral hospitals. One hundred and forty-three (143) bacteriological samples were collected for analysis and fifty bacterial isolates were obtained which consist of 13 (26\%) and 37 (74\%) bacterial isolates from air sample and surface swabs respectively (Figure 2). The selected surfaces comprise bed rails, doorknobs, floor, nurse table top, operation tables, sinks, stretchers and toilet seats. The bacteriological assessment of the indoor air in different wards and the selected surfaces is shown in Figure 3. Thus, bacteria isolated from hospital air include 13 (26\%), bed rails 8 (16\%), door knobs 1 (2\%), floor 2 (4\%), nurse table top 5 (10\%), operation table 2 (4\%), sink 9 (18\%), stretchers 1 (2\%) and toilet seat 6 (12\%). The cultural, morphological and biochemical characteristics of the bacterial isolates are shown in Table $\mathbf{1}$ as described in Bergey's Manual 9th edition (Bergey, 2000).

A total of fifty bacterial were isolated from the threereferral hospital viz: City Hospital 13 (26\%), Federal Medical Centre Makurdi 20 (40\%) and Saint Theresa Hospital 17 (34\%) which comprises of 17 (34\%) S. aureus, 9 (18\%) S. spp, 9 (18\%) P. aeruginosa, 4 (8\%) E. coli and 5 (10\%) Klebsiella spp (Table 2 and 3$)$. The bacteria isolates involved in this work are potential 
Table 1. Morphological and Biochemical Characteristics of Bacterial Isolates

\begin{tabular}{|c|c|c|c|c|c|c|c|c|c|c|c|c|c|c|c|c|c|}
\hline $\begin{array}{l}\text { Isolate } \\
\text { code }\end{array}$ & $\begin{array}{c}\text { Cultural } \\
\text { morphology } \\
\text { on nutrient agar }\end{array}$ & 㝕 & 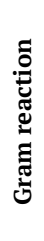 & 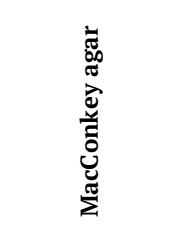 & 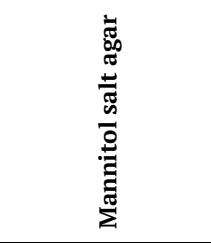 & 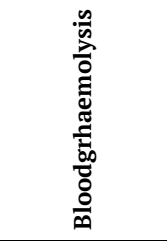 & $\frac{\ddot{\Xi}}{\frac{\pi}{\pi}}$ & 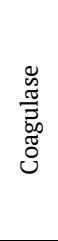 & $\begin{array}{l}\frac{0}{\circ} \\
\stackrel{0}{\Xi}\end{array}$ & 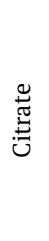 & 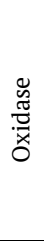 & 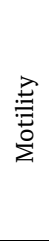 & 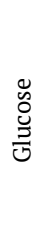 & 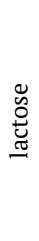 & 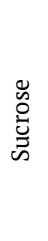 & 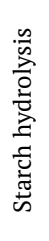 & Identification \\
\hline $\mathbf{S B R}_{2}$ & $\begin{array}{c}\text { Opaque, milky and } \\
\text { golden yellowish } \\
\text { colour }\end{array}$ & $\begin{array}{l}\text { Coccci in } \\
\text { clusters }\end{array}$ & + & No growth & $\begin{array}{l}\text { Growth, mannitol } \\
\text { fermented }\end{array}$ & $\beta$-haemolysis & + & + & - & + & - & - & + & + & + & + & $\begin{array}{c}\text { Staphylococcus } \\
\text { aureus }\end{array}$ \\
\hline $\mathrm{STs}_{1}$ & $\begin{array}{l}\text { Flat growth with } \\
\text { greenish colour and } \\
\text { offensive odour }\end{array}$ & Rods & - & $\begin{array}{l}\text { Flat growth with } \\
\text { an offensive } \\
\text { odour }\end{array}$ & ND & $\beta$-haemolysis & + & - & - & - & + & + & - & - & - & - & $\begin{array}{l}\text { Pseudomonas } \\
\text { aeruginosa }\end{array}$ \\
\hline $\mathrm{SNT}_{2}$ & $\begin{array}{l}\text { Flat growth with } \\
\text { greenish colour and } \\
\text { offensive odour }\end{array}$ & Rods & & $\begin{array}{l}\text { Flat growth with } \\
\text { an offensive } \\
\text { odour } \\
\end{array}$ & ND & $\beta$-haemolysis & + & - & - & - & + & + & - & - & - & - & $\begin{array}{l}\text { Pseudomonas } \\
\text { aeruginosa }\end{array}$ \\
\hline STS $_{2}$ & $\begin{array}{c}\text { Opaque, milky and } \\
\text { golden yellowish } \\
\text { colour }\end{array}$ & $\begin{array}{l}\text { Coccci in } \\
\text { clusters }\end{array}$ & + & No growth & $\begin{array}{l}\text { Growth, mannitol } \\
\text { fermented }\end{array}$ & $\beta$-haemolysis & + & + & - & + & - & - & + & + & + & + & $\begin{array}{c}\text { Staphylococcus } \\
\text { aureus }\end{array}$ \\
\hline $\mathrm{SNT}_{4}$ & $\begin{array}{l}\text { Opaque and milky } \\
\text { growth }\end{array}$ & $\begin{array}{l}\text { Coccci in } \\
\text { clusters }\end{array}$ & + & No growth & $\begin{array}{c}\text { Growth, mannitol } \\
\text { not fermented }\end{array}$ & $\alpha$-haemolysis & + & - & - & - & - & - & + & + & + & - & $\begin{array}{c}\text { Staphylococcus } \\
\text { spp }\end{array}$ \\
\hline $\mathrm{SSK}_{4}$ & $\begin{array}{l}\text { Opaque and milky } \\
\text { growth }\end{array}$ & $\begin{array}{l}\text { Coccci in } \\
\text { clusters }\end{array}$ & + & No growth & $\begin{array}{c}\text { Growth, mannitol } \\
\text { not fermented }\end{array}$ & $\alpha$-haemolysis & + & - & - & - & - & - & + & + & + & - & $\begin{array}{c}\text { Staphylococcus } \\
\text { spp } \\
\end{array}$ \\
\hline SSK $_{1}$ & $\begin{array}{l}\text { Opaque and milky } \\
\text { growth }\end{array}$ & $\begin{array}{l}\text { Coccci in } \\
\text { clusters }\end{array}$ & + & No growth & $\begin{array}{c}\text { Growth, mannitol } \\
\text { not fermented }\end{array}$ & $\beta$-haemolysis & + & - & - & - & - & - & + & + & + & - & $\begin{array}{c}\text { Staphylococcus } \\
\text { spp } \\
\end{array}$ \\
\hline $\mathrm{SBR}_{3}$ & $\begin{array}{l}\text { Opaque and milky } \\
\text { growth }\end{array}$ & $\begin{array}{l}\text { Coccci in } \\
\text { clusters }\end{array}$ & + & No growth & $\begin{array}{c}\text { Growth, mannitol } \\
\text { not fermented }\end{array}$ & $\alpha$-haemolysis & + & - & - & - & - & - & + & + & + & - & $\begin{array}{c}\text { Staphylococcus } \\
\text { spp } \\
\end{array}$ \\
\hline $\mathrm{SBR}_{5}$ & $\begin{array}{c}\text { Opaque, milky and } \\
\text { golden yellowish } \\
\text { colour }\end{array}$ & $\begin{array}{l}\text { Coccci in } \\
\text { clusters }\end{array}$ & + & No growth & $\begin{array}{l}\text { Growth, mannitol } \\
\text { fermented }\end{array}$ & $\beta$-haemolysis & + & + & - & + & - & - & + & + & + & + & $\begin{array}{c}\text { Staphylococcus } \\
\text { aureus }\end{array}$ \\
\hline $\mathrm{SFL}_{3}$ & $\begin{array}{l}\text { Convex and entire } \\
\text { growth }\end{array}$ & Short rods & - & Growth & ND & ND & + & ND & + & - & - & + & + & + & - & - & $\begin{array}{l}\text { Escherichia } \\
\text { coli }\end{array}$ \\
\hline $\mathrm{SFL}_{2}$ & $\begin{array}{c}\text { Convex and entire } \\
\text { growth } \\
\end{array}$ & Short rods & - & Growth & ND & ND & + & ND & + & - & - & + & + & + & - & - & $\begin{array}{c}\text { Escherichia } \\
\text { coli }\end{array}$ \\
\hline $\mathrm{SFL}_{1}$ & $\begin{array}{c}\text { Opaque, milky and } \\
\text { golden yellowish } \\
\text { colour }\end{array}$ & $\begin{array}{l}\text { Coccci in } \\
\text { clusters }\end{array}$ & + & No growth & $\begin{array}{l}\text { Growth, mannitol } \\
\text { fermented }\end{array}$ & $\beta$-haemolysis & + & + & - & + & - & - & + & + & + & + & $\begin{array}{c}\text { Staphylococcus } \\
\text { aureus }\end{array}$ \\
\hline SPD $\mathrm{AR}_{2}$ & $\begin{array}{l}\text { Flat growth with } \\
\text { greenish colour and } \\
\text { offensive odour }\end{array}$ & Rods & & $\begin{array}{l}\text { Flat growth with } \\
\text { an offensive } \\
\text { odour }\end{array}$ & ND & $\beta$-haemolysis & + & - & - & - & + & + & - & - & - & - & $\begin{array}{l}\text { Pseudomonas } \\
\text { aeruginosa }\end{array}$ \\
\hline SPD $\mathrm{AR}_{3}$ & $\begin{array}{c}\text { Opaque, milky and } \\
\text { golden yellowish } \\
\text { colour }\end{array}$ & $\begin{array}{l}\text { Coccci in } \\
\text { clusters }\end{array}$ & + & No growth & $\begin{array}{l}\text { Growth, mannitol } \\
\text { fermented }\end{array}$ & $\beta$-haemolysis & + & + & - & + & - & - & + & + & + & + & $\begin{array}{c}\text { Staphylococcus } \\
\text { aureus }\end{array}$ \\
\hline SMW $\mathbf{A R}_{2}$ & $\begin{array}{c}\text { Convex and entire } \\
\text { growth }\end{array}$ & Short rods & - & Growth & ND & ND & + & ND & + & - & - & + & + & + & - & - & $\begin{array}{c}\text { Escherichia } \\
\text { coli }\end{array}$ \\
\hline $\mathrm{SMW} \mathrm{AR}_{3}$ & $\begin{array}{c}\text { Convex and entire } \\
\text { growth } \\
\end{array}$ & Short rods & - & Growth & ND & ND & + & ND & + & - & - & + & + & + & - & - & $\begin{array}{c}\text { Escherichia } \\
\text { coli }\end{array}$ \\
\hline STH DK 2 & $\begin{array}{c}\text { Opaque, milky and } \\
\text { golden yellowish } \\
\text { colour }\end{array}$ & $\begin{array}{l}\text { Coccci in } \\
\text { clusters }\end{array}$ & + & No growth & $\begin{array}{l}\text { Growth, mannitol } \\
\text { fermented }\end{array}$ & $\beta$-haemolysis & + & + & - & + & - & - & + & + & + & + & $\begin{array}{c}\text { Staphylococcus } \\
\text { aureus }\end{array}$ \\
\hline $\mathrm{CMwAR}_{5}$ & $\begin{array}{c}\text { Opaque, milky and } \\
\text { golden yellowish } \\
\text { colour }\end{array}$ & $\begin{array}{l}\text { Coccci in } \\
\text { clusters }\end{array}$ & + & No growth & $\begin{array}{l}\text { Growth, mannitol } \\
\text { fermented }\end{array}$ & $\beta$-haemolysis & + & + & - & + & - & - & + & + & + & + & $\begin{array}{c}\text { Staphylococcus } \\
\text { aureus }\end{array}$ \\
\hline $\mathrm{CST}_{1}$ & $\begin{array}{c}\text { Opaque, milky and } \\
\text { golden yellowish } \\
\text { colour }\end{array}$ & $\begin{array}{l}\text { Coccci in } \\
\text { clusters }\end{array}$ & + & No growth & $\begin{array}{l}\text { Growth, mannitol } \\
\text { fermented }\end{array}$ & $\beta$-haemolysis & + & + & - & + & - & - & + & + & + & + & $\begin{array}{c}\text { Staphylococcus } \\
\text { aureus }\end{array}$ \\
\hline $\mathrm{CBR}_{2}$ & $\begin{array}{l}\text { Flat growth with } \\
\text { greenish colour and } \\
\text { offensive odour }\end{array}$ & Rods & - & $\begin{array}{l}\text { Flat growth with } \\
\text { an offensive } \\
\text { odour } \\
\end{array}$ & ND & $\beta$-haemolysis & + & - & - & - & + & + & - & - & - & - & $\begin{array}{l}\text { Pseudomonas } \\
\text { aeruginosa }\end{array}$ \\
\hline $\mathbf{C F L}_{2}$ & $\begin{array}{c}\text { Opaque, milky and } \\
\text { golden yellowish } \\
\text { colour }\end{array}$ & $\begin{array}{l}\text { Coccciin } \\
\text { clusters }\end{array}$ & + & No growth & $\begin{array}{l}\text { Growth, mannitol } \\
\text { fermented }\end{array}$ & $\beta$-haemolysis & + & + & - & + & - & - & + & + & + & + & $\begin{array}{c}\text { Staphylococcus } \\
\text { aureus }\end{array}$ \\
\hline $\mathrm{CNT}_{3}$ & $\begin{array}{l}\text { Convex and entire } \\
\text { growth }\end{array}$ & Short rods & - & Growth & ND & ND & + & ND & + & - & - & + & + & + & - & - & $\begin{array}{l}\text { Escherichia } \\
\text { coli }\end{array}$ \\
\hline $\mathrm{CTS}_{2}$ & $\begin{array}{c}\text { Convex and entire } \\
\text { growth }\end{array}$ & $\begin{array}{l}\text { Coccci in } \\
\text { clusters }\end{array}$ & + & No growth & $\begin{array}{c}\text { Growth, mannitol } \\
\text { not fermented }\end{array}$ & $\alpha$-haemolysis & + & - & - & - & - & - & + & + & + & - & $\begin{array}{c}\text { Staphylococcus } \\
\text { spp }\end{array}$ \\
\hline $\operatorname{coT}_{1}$ & $\begin{array}{c}\text { Opaque, milky and } \\
\text { golden yellowish } \\
\text { colour }\end{array}$ & $\begin{array}{l}\text { Coccci in } \\
\text { clusters }\end{array}$ & + & No growth & $\begin{array}{l}\text { Growth, mannitol } \\
\text { fermented }\end{array}$ & $\beta$-haemolysis & + & + & - & + & - & - & + & + & + & + & $\begin{array}{c}\text { Staphylococcus } \\
\text { aureus }\end{array}$ \\
\hline $\mathrm{COT}_{4}$ & $\begin{array}{c}\text { Opaque, milky and } \\
\text { golden yellowish } \\
\text { colour }\end{array}$ & $\begin{array}{l}\text { Coccci in } \\
\text { clusters }\end{array}$ & + & No growth & $\begin{array}{l}\text { Growth, mannitol } \\
\text { fermented }\end{array}$ & $\beta$-haemolysis & + & + & - & + & - & - & + & + & + & + & $\begin{array}{c}\text { Staphylococcus } \\
\text { aureus }\end{array}$ \\
\hline
\end{tabular}

Key: +: Positive -: Negative ND: Not determined 
Table 1 (continued). Morphological and Biochemical Characteristics of Bacterial Isolates

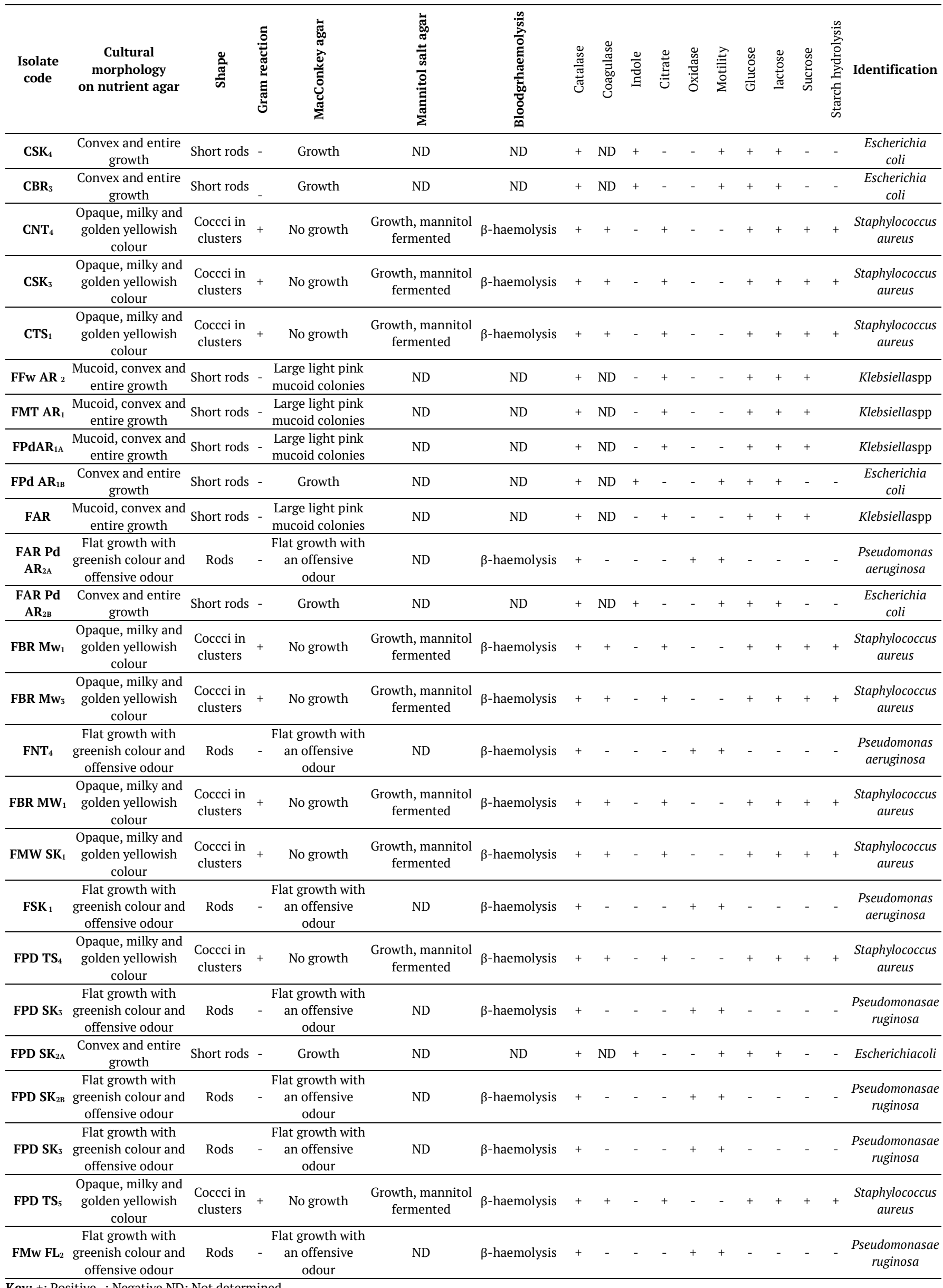

Key: +: Positive -: Negative ND: Not determined 
Table 2. Incidence of Bacterial Isolates in Relation to Sample Source

\begin{tabular}{|c|c|c|c|c|c|c|}
\hline $\begin{array}{l}\text { Sample } \\
\text { Source }\end{array}$ & $\begin{array}{c}\text { Total positive } \\
\text { Isolates (\%) }\end{array}$ & $\begin{array}{c}\text { Pseudomonas } \\
\text { aeruginosa }\end{array}$ & $\begin{array}{l}\text { Klebsiella } \\
\text { spp }\end{array}$ & $\begin{array}{c}\text { Escherichia } \\
\text { coli }\end{array}$ & $\begin{array}{c}\text { Staphylococcus } \\
\text { Aureus }\end{array}$ & $\begin{array}{c}\text { Staphylococcus } \\
\text { spp }\end{array}$ \\
\hline $\mathrm{AR}$ & $13(18.3)$ & $1(18.2)$ & $5(100)$ & $4(36.4)$ & $3(5.1)$ & $0(0)$ \\
\hline $\mathrm{BR}$ & $8(11.3)$ & $1(12.5)$ & $0(0)$ & $1(9.1)$ & $4(12.8)$ & $2(16.7)$ \\
\hline DK & $1(1.4)$ & $0(0)$ & $0(0)$ & $0(0)$ & $1(2.6)$ & $0(0)$ \\
\hline FL & $5(7.0)$ & $1(9.1)$ & $0(0)$ & $2(18.2)$ & $2(5.1)$ & $0(0)$ \\
\hline NT & $5(7.0)$ & $2(18.2)$ & $0(0)$ & $1(9.1)$ & $1(2.6)$ & $1(16.7)$ \\
\hline SK & $9(12.7)$ & $3(27.3)$ & $0(0)$ & $2(18.2)$ & $2(7.7)$ & $2(16.7)$ \\
\hline ST & $1(1.4)$ & $0(0)$ & $0(0)$ & $0(0)$ & $1(2.6)$ & $0(0)$ \\
\hline OT & $2(2.8)$ & $0(0)$ & $0(0)$ & $0(0)$ & $2(5.1)$ & $0(0)$ \\
\hline TS & $6(8.5)$ & $1(9.1)$ & $0(0)$ & $0(0)$ & $4(10.3)$ & $1(16.7)$ \\
\hline Total & $50(100)$ & $9(100)$ & $5(100)$ & 11(100) & 36100) & $10(100)$ \\
\hline
\end{tabular}

AR- Air Sample, BR- Bed Rails, DK- Door knobs, ST- Stretchers, SK-Sink, TS - Toilet seat, OT - Operation table, FL - Floor, TS - Toilet Seat, NT Nurse Table Top

Table 3. Incidence of Bacterial Isolates in Hospitals from the Three Referral Hospitals

\begin{tabular}{cccccc}
\hline Hospitals & Staphylococcus aureus & $\begin{array}{c}\text { Staphylococcus } \\
\text { spp }\end{array}$ & $\begin{array}{c}\text { Pseudomonas } \\
\text { aeruginosa }\end{array}$ & Escherichia coli & $\begin{array}{c}\text { Klebsiella } \\
\text { spp }\end{array}$ \\
\hline CHM & $8(16 \%)$ & $1(2 \%)$ & $1(2 \%)$ & $3(6 \%)$ & $0(0)$ \\
\hline FMC & $2(4 \%)$ & $4(8 \%)$ & $6(12 \%)$ & $3(6 \%)$ & $5(100)$ \\
\hline STH & $7(14 \%)$ & $4(8 \%)$ & $2(4 \%)$ & $4(8 \%)$ & $0(0)$ \\
\hline Total & $\mathbf{1 7}(\mathbf{3 4 \% )}$ & $\mathbf{9 ( 1 8 \% )}$ & $\mathbf{9 ( 1 8 \% )}$ & $\mathbf{1 0}(\mathbf{2 0 \% )}$ & $\mathbf{5 ( 1 0 \% )}$ \\
\hline
\end{tabular}

Key: CH- City Hospital, FMCW- Federal Medical Centre Wadata, STH - Saint Theresa Hospital Figures in brackets are the percentage rates of occurrence of the bacterial isolates

cause of nosocomial infections. This is in agreement with the findings of Bereket et al. (2012) which revealed genus Staphylococcus, Escherichia coli and Pseudomonas aeruginosa as commonly isolated nosocomial pathogens. There is lower incidence of Gram-negative bacterial isolates 15 (30\%) than Gram positive bacterial isolates 35 (70\%), which suggests that most of the bacterial are from human source (Prescott, Harley and Klein, 2008).

The Gram-negative bacteria are Klebsiella spp (10\%), Pseudomonas aeruginosa (18\%) and Escherichia coli (20\%). This is also in agreement with findings by Hassan, Aftab and Riffat, (2015) that, 3-7\% of nosocomial bacterial infections are related to Klebsiella pneumonia. Klebsiella is a Gram-negative, nonmotile, encapsulated, rod-shaped bacillus present within the nasopharynx and gastrointestinal tract of humans and nonhuman primates. E. coli is one of the common Gramnegative bacteria often associated with wound sepsis and endotoxin-induced shock (Hassan, Aftab and Riffat, 2015). It often causes urinary tract and wound infections in patient with compromised immunity. Drach (2005) revealed Klebsiella spp and E. coli among others as the microorganisms related to urinary catheterization. Bereket et al. (2012) reported that Pseudomonas aeruginosa can transiently colonize the respiratory and gastrointestinal tracts of hospitalized individuals. Furthermore, giving the preponderance of these organisms in the present study, there is a high possibility of patients contracting such infections. According to Brooks et al. (2010), Klebsiella can cause respiratory tract infection, urinary tract infections as well as wounds infections in a poor ventilated environment.

The percentage of occurrence of Staphylococcus aureus were (34\%) and other Staphylococcuss spp were (18\%). Out of 26 (Twenty-six) Gram positive cocci isolated, Staphylococcus aureus were 17 (65.4\%) while Staphylococcus spp were 9 (34.6\%). This report agrees with the findings by Hassan, Aftab and Riffat (2015) that Staphylococcus spp had the least occurrence among the Gram-positive bacteria associated with hospital acquired infections. Staphylococcus aureus being among normal microbiota of human may be responsible for higher number of incidence (65.4\%).

Therefore, the nine sources in this study which include air and other eight selected surfaces shown in Figure 3 and Table 3 are multi-reservoirs being responsible for hospital contamination. This was also the same with the findings of Wilks, Michels and Keevil (2005) and Michels (2006) that inanimate objects can allow survival of microorganisms for a particular period of time. However, this work is in contrast to a report by Derese et al. (2016) where the main isolates in a similar finding executed in selected wards of a referral hospital in Ethiopia were coagulase negative Staphylococci (CoNs) 44\%, followed by $S$. aureus, $37.4 \%$, and Klebsiella spp at $11.6 \%$.

Tables 4-6 shows antibiotics susceptibility pattern of the bacteria isolates from the three hospitals. 28 (56\%) bacterial isolates were resistant to almost all the antibiotics employed in the study. $P$. aeruginosa showed highest resistant to the antibiotics considered in the three referral hospitals. The least incidence of Klebsiella spp in this finding from air sample of one of the three locations affirms the report of Sani et al. (2012) which stated that Klebsiella spp was the least commonly isolated bacteria among the Gram-negative facultative Bacillus despite the sample source differs. This shows consistency to previous findings on microbiological assessment of indoor and outdoor air quality in a general hospital in north-east Nigeria. The factors which encourage the growth and multiplication of airborne microbes need to be controlled in the Hospital environment to reduce the rate of nosocomial infections (Agwaranze et al., 2020). 
Table 4. Zones of inhibition for Bacterial isolates from City Hospital

\begin{tabular}{|c|c|c|c|c|c|c|c|c|c|}
\hline \multirow{2}{*}{ Isolate code } & \multicolumn{8}{|c|}{ Antibiotics measured in millimeter } & \multirow{2}{*}{ Bacteria } \\
\hline & AUG & CAZ & CRX & CTR & CXC & ERY & GEN & OFL & \\
\hline $\mathrm{CMwAR}_{5}$ & $35(\mathrm{~s})$ & $0(\mathrm{r})$ & $22(\mathrm{~s})$ & $27(\mathrm{~s})$ & $26(s)$ & $0(\mathrm{r})$ & $0(\mathrm{r})$ & $20(s)$ & Staphylococcus aureus \\
\hline $\mathrm{CST}_{1}$ & $0(\mathrm{r})$ & $0(\mathrm{r})$ & $0(\mathrm{r})$ & $0(\mathrm{r})$ & $0(\mathrm{r})$ & $0(\mathrm{r})$ & $0(\mathrm{r})$ & $25(\mathrm{~s})$ & Staphylococcus aureus \\
\hline $\mathrm{CBR}_{2}$ & $0(\mathrm{r})$ & $0(\mathrm{r})$ & $0(\mathrm{r})$ & $0(\mathrm{r})$ & $0(\mathrm{r})$ & $0(\mathrm{r})$ & $0(\mathrm{r})$ & $0(\mathrm{r})$ & Pseudomonas aeruginosa \\
\hline $\mathrm{CFL}_{2}$ & $32(\mathrm{~s})$ & $0(\mathrm{r})$ & $30(\mathrm{~s})$ & $30(\mathrm{~s})$ & $0(\mathrm{r})$ & $0(\mathrm{r})$ & $30(\mathrm{~s})$ & $23(\mathrm{~s})$ & Staphylococcus aureus \\
\hline $\mathrm{CNT}_{3}$ & $33(\mathrm{~s})$ & $0(\mathrm{r})$ & $10(\mathrm{r})$ & $25(\mathrm{~s})$ & $0(\mathrm{r})$ & $0(\mathrm{r})$ & $0(\mathrm{r})$ & $0(\mathrm{r})$ & Escherichia coli \\
\hline CTS $_{2}$ & $30(\mathrm{~s})$ & $0(\mathrm{r})$ & $26(s)$ & $26(s)$ & $0(\mathrm{r})$ & $34(\mathrm{~s})$ & $0(\mathrm{r})$ & $0(\mathrm{r})$ & Staphylococcus spp \\
\hline $\mathrm{COT}_{1}$ & $0(\mathrm{r})$ & $0(\mathrm{r})$ & $22(\mathrm{~s})$ & $22(s)$ & $0(\mathrm{r})$ & $0(\mathrm{r})$ & $22(s)$ & $0(\mathrm{r})$ & Staphylococcus aureus \\
\hline $\mathrm{COT}_{4}$ & $0(\mathrm{r})$ & $0(\mathrm{r})$ & $0(\mathrm{r})$ & $0(\mathrm{r})$ & $0(\mathrm{r})$ & $0(\mathrm{r})$ & $0(\mathrm{r})$ & $24(\mathrm{~s})$ & Staphylococcus aureus \\
\hline $\mathrm{CSK}_{4}$ & $0(\mathrm{r})$ & $0(\mathrm{r})$ & $0(\mathrm{r})$ & $0(\mathrm{r})$ & $0(\mathrm{r})$ & $0(\mathrm{r})$ & $25(\mathrm{~s})$ & $28(s)$ & Escherichia coli \\
\hline $\mathrm{CBR}_{3}$ & $24(\mathrm{~s})$ & $0(\mathrm{r})$ & $24(s)$ & $0(\mathrm{r})$ & $27(s)$ & $33(\mathrm{~s})$ & $0(\mathrm{r})$ & $25(\mathrm{~s})$ & Escherichia coli \\
\hline $\mathrm{CNT}_{4}$ & $35(\mathrm{~s})$ & $0(\mathrm{r})$ & $22(s)$ & $27(s)$ & $26(s)$ & $0(\mathrm{r})$ & $0(\mathrm{r})$ & $20(s)$ & Staphylococcus aureus \\
\hline $\mathrm{CSK}_{3}$ & $24(\mathrm{~s})$ & $24(\mathrm{~s})$ & $26(\mathrm{~s})$ & $26(\mathrm{~s})$ & $20(\mathrm{~s})$ & $26(\mathrm{~s})$ & $25(\mathrm{~s})$ & $22(\mathrm{~s})$ & Staphylococcus aureus \\
\hline CTS $_{1}$ & $0(\mathrm{r})$ & $0(\mathrm{r})$ & $0(\mathrm{r})$ & $0(\mathrm{r})$ & $0(\mathrm{r})$ & $0(\mathrm{r})$ & $0(\mathrm{r})$ & $25(\mathrm{~s})$ & Staphylococcus aureus \\
\hline
\end{tabular}

Key: C- City Hospital, NT- Nurse Table Top, BR- Bed Rails, DK- Door knobs, ST-Stretchers, SK- Sink,TS- Toilet seat, OT- Operation table, FL- Floor, TS -

Toilet Seat, AR- Hospital air, $r=$ resistant, $\mathrm{s}=$ sensitive

Table 5. Zones of inhibition for Bacterial isolates from Federal Medical Centre, Wadata

\begin{tabular}{|c|c|c|c|c|c|c|c|c|c|}
\hline \multirow{2}{*}{ Isolate code } & \multicolumn{8}{|c|}{ Antibiotics measured in millimeter } & \multirow{2}{*}{ Bacteria } \\
\hline & AUG & CAZ & CRX & CTR & CXC & ERY & GEN & OFL & \\
\hline FFw AR $_{2}$ & $35(\mathrm{~s})$ & $30(\mathrm{~s})$ & $30(\mathrm{~s})$ & $28(\mathrm{~s})$ & $8(\mathrm{r})$ & $8(\mathrm{r})$ & $28(\mathrm{~s})$ & $30(\mathrm{~s})$ & Klebsiella spp \\
\hline FMT AR ${ }_{1}$ & $30(\mathrm{~s})$ & $30(\mathrm{~s})$ & $34(\mathrm{~s})$ & $30(\mathrm{~s})$ & $6(\mathrm{r})$ & $8(\mathrm{r})$ & $20(\mathrm{~s})$ & $30(\mathrm{~s})$ & Klebsiella spp \\
\hline FPd $\mathrm{AR}_{1 \mathrm{~A}}$ & $26(\mathrm{~s})$ & $26(\mathrm{~s})$ & $36(\mathrm{~s})$ & $27(\mathrm{~s})$ & $12(\mathrm{r})$ & $8(\mathrm{r})$ & $34(\mathrm{~s})$ & $36(\mathrm{~s})$ & Klebsiellas pp \\
\hline FPd $\mathrm{AR}_{1 \mathrm{~B}}$ & $28(\mathrm{~s})$ & $36(\mathrm{~s})$ & $36(\mathrm{~s})$ & $34(\mathrm{~s})$ & $12(\mathrm{r})$ & $10(\mathrm{r})$ & $26(\mathrm{~s})$ & $38(\mathrm{~s})$ & Escherichia coli \\
\hline FAR & $8(\mathrm{r})$ & $8(\mathrm{r})$ & $8(\mathrm{r})$ & $8(\mathrm{r})$ & $8(\mathrm{r})$ & $8(\mathrm{r})$ & $8(\mathrm{r})$ & $8(\mathrm{r})$ & Klebsiella spp \\
\hline FAR Pd $\mathrm{AR}_{2 \mathrm{~A}}$ & $0(\mathrm{r})$ & $0(\mathrm{r})$ & $0(\mathrm{r})$ & $0(\mathrm{r})$ & $0(\mathrm{r})$ & $0(\mathrm{r})$ & $0(\mathrm{r})$ & $0(\mathrm{r})$ & Pseudomonas aeruginosa \\
\hline FAR Pd $\mathrm{AR}_{2 \mathrm{~B}}$ & $32(\mathrm{~s})$ & $6(\mathrm{r})$ & $36(\mathrm{~s})$ & $6(\mathrm{r})$ & $6(\mathrm{r})$ & $6(r)$ & $18(\mathrm{~s})$ & $32(\mathrm{~s})$ & Escherichia coli \\
\hline FBR Mw & $28(\mathrm{~s})$ & $26(\mathrm{~s})$ & $32(\mathrm{~s})$ & $6(r)$ & $6(r)$ & $6(r)$ & $24(\mathrm{~s})$ & $30(\mathrm{~s})$ & Staphylococcus aureus \\
\hline FBR Mw3 & $4(\mathrm{r})$ & $4(r)$ & $4(r)$ & $4(\mathrm{r})$ & $4(\mathrm{r})$ & $4(r)$ & $4(\mathrm{r})$ & $4(\mathrm{r})$ & Staphylococcus aureus \\
\hline $\mathrm{FNT}_{4}$ & $4(\mathrm{r})$ & $4(r)$ & $4(r)$ & $4(\mathrm{r})$ & $4(r)$ & $4(r)$ & $4(\mathrm{r})$ & $4(\mathrm{r})$ & Pseudomonas aeruginosa \\
\hline FBR MW & $5(\mathrm{r})$ & $5(\mathrm{r})$ & $5(\mathrm{r})$ & $5(\mathrm{r})$ & $5(\mathrm{r})$ & $5(\mathrm{r})$ & $5(\mathrm{r})$ & $5(\mathrm{r})$ & Staphylococcus aureus \\
\hline FMW SK $_{1}$ & $6(r)$ & $6(r)$ & $6(\mathrm{r})$ & $34(\mathrm{~s})$ & $6(r)$ & $6(r)$ & $6(r)$ & $34(\mathrm{~s})$ & Staphylococcus aureus \\
\hline FPD SK $_{1}$ & $6(r)$ & $6(\mathrm{r})$ & $6(\mathrm{r})$ & $18(\mathrm{~s})$ & $6(\mathrm{r})$ & $6(\mathrm{r})$ & $6(\mathrm{r})$ & $24(\mathrm{~s})$ & Pseudomonas aeruginosa \\
\hline FPD TS $\mathrm{TS}_{4}$ & $28(\mathrm{~s})$ & $24(\mathrm{~s})$ & $28(\mathrm{~s})$ & $6(\mathrm{r})$ & $8(\mathrm{r})$ & $12(\mathrm{r})$ & $22(\mathrm{~s})$ & $28(\mathrm{~s})$ & Staphylococcus aureus \\
\hline FPD SK $_{3}$ & $18(\mathrm{~s})$ & $5(\mathrm{r})$ & $26(\mathrm{~s})$ & $28(\mathrm{~s})$ & $5(\mathrm{r})$ & $5(r)$ & $5(\mathrm{r})$ & $34(\mathrm{~s})$ & Pseudomonas aeruginosa \\
\hline FPD SK $_{2 \mathrm{~A}}$ & $16(\mathrm{i})$ & $6(\mathrm{r})$ & $6(\mathrm{r})$ & $32(\mathrm{~s})$ & $6(r)$ & $6(r)$ & $6(r)$ & $30(\mathrm{~s})$ & Escherichia coli \\
\hline FPD SK $_{2 B}$ & $4(\mathrm{r})$ & $4(r)$ & $4(r)$ & $4(\mathrm{r})$ & $4(\mathrm{r})$ & $4(r)$ & $4(\mathrm{r})$ & $4(\mathrm{r})$ & Pseudomonas aeruginosa \\
\hline FPD SK $_{3}$ & $6(r)$ & $6(r)$ & $6(\mathrm{r})$ & $6(r)$ & $6(r)$ & $6(r)$ & $6(r)$ & $18(\mathrm{~s})$ & Pseudomonas aeruginosa \\
\hline FPD TS ${ }_{5}$ & $22(\mathrm{~s})$ & $(0) \mathrm{r}$ & $(0) \mathrm{r}$ & $18(\mathrm{~s})$ & $6(r)$ & $6(r)$ & $6(r)$ & $20(\mathrm{~s})$ & Staphylococcus aureus \\
\hline FMw $\mathrm{FL}_{2}$ & $8(\mathrm{r})$ & $8(\mathrm{r})$ & $28(\mathrm{~s})$ & $22(\mathrm{~s})$ & $8(r)$ & $8(r)$ & $8(r)$ & $28(\mathrm{~s})$ & Pseudomonas aeruginosa \\
\hline
\end{tabular}

Key: F- Federal Medical Centre Wadata, NT- Nurse Table Top, BR- Bed Rails, DK- Door knobs, ST-Stretchers, SK- Sink, TS- Toilet seat, OT- Operation table, FL- Floor, TS - Toilet Seat, AR- Hospital air, $r=$ resistant, $\mathrm{s}=$ sensitive

Table 6. Zones of Inhibition for Bacterial Isolates from Saint Theresa Hospital

\begin{tabular}{|c|c|c|c|c|c|c|c|c|c|}
\hline \multirow{2}{*}{ Isolate code } & \multicolumn{8}{|c|}{ Antibiotics measured in millimeter } & \multirow[b]{2}{*}{ Bacteria } \\
\hline & AUG & CAZ & CRX & CTR & CXC & ERY & GEN & OFL & \\
\hline $\mathrm{SBR}_{2}$ & $31(\mathrm{~s})$ & $0(\mathrm{r})$ & $20(s)$ & $12(\mathrm{r})$ & $12(\mathrm{r})$ & $12(\mathrm{r})$ & $26(s)$ & $27(s)$ & Staphylococcus aureus \\
\hline STs 1 & $0(\mathrm{r})$ & $0(\mathrm{r})$ & $0(\mathrm{r})$ & $0(\mathrm{r})$ & $0(\mathrm{r})$ & $0(\mathrm{r})$ & $0(\mathrm{r})$ & $0(\mathrm{r})$ & Pseudomonas aeruginosa \\
\hline $\mathrm{SNT}_{2}$ & $0(\mathrm{r})$ & $8(\mathrm{r})$ & $0(\mathrm{r})$ & $0(\mathrm{r})$ & $0(\mathrm{r})$ & $9(\mathrm{r})$ & $18(\mathrm{~s})$ & $20(\mathrm{~s})$ & Pseudomonas aeruginosa \\
\hline STS $_{2}$ & $27(\mathrm{~s})$ & $0(\mathrm{r})$ & $0(\mathrm{r})$ & $20(\mathrm{~s})$ & $15(\mathrm{i})$ & $0(\mathrm{r})$ & $14(\mathrm{i})$ & $0(\mathrm{r})$ & Staphylococcus aureus \\
\hline $\mathrm{SNT}_{4}$ & $28(\mathrm{~s})$ & $0(\mathrm{r})$ & $0(\mathrm{r})$ & $0(\mathrm{r})$ & $0(\mathrm{r})$ & $24(\mathrm{~s})$ & $26(s)$ & $24(s)$ & Staphylococcus spp \\
\hline SSK $_{4}$ & $28(\mathrm{~s})$ & $0(\mathrm{r})$ & $27(\mathrm{~s})$ & $18(\mathrm{~s})$ & $17(\mathrm{~s})$ & $0(\mathrm{r})$ & $27(\mathrm{~s})$ & $20(s)$ & Staphylococcus spp \\
\hline SSK $_{1}$ & $0(\mathrm{r})$ & $0(\mathrm{r})$ & $0(\mathrm{r})$ & $0(\mathrm{r})$ & $0(\mathrm{r})$ & $25(\mathrm{~s})$ & $0(\mathrm{r})$ & $29(\mathrm{~s})$ & Staphylococcus spp \\
\hline $\mathrm{SBR}_{3}$ & $15(\mathrm{i})$ & $0(\mathrm{r})$ & $28(\mathrm{~s})$ & $23(s)$ & $0(\mathrm{r})$ & $22(\mathrm{~s})$ & $30(\mathrm{r})$ & $0(\mathrm{r})$ & Staphylococcus spp \\
\hline SBR $_{5}$ & $0(\mathrm{r})$ & $0(\mathrm{r})$ & $18(\mathrm{~s})$ & $25(\mathrm{r})$ & $0(\mathrm{r})$ & $0(\mathrm{r})$ & $0(\mathrm{r})$ & $25(\mathrm{~s})$ & Staphylococcus aureus \\
\hline $\mathrm{SFL}_{3}$ & $25(\mathrm{~s})$ & $0(\mathrm{r})$ & $0(\mathrm{r})$ & $25(\mathrm{~s})$ & $0(r)$ & $30(\mathrm{~s})$ & $20(s)$ & 16(i) & Escherichia coli \\
\hline $\mathrm{SFL}_{2}$ & $0(\mathrm{r})$ & $16(\mathrm{i})$ & $0(\mathrm{r})$ & $12(\mathrm{r})$ & $0(\mathrm{r})$ & $0(\mathrm{r})$ & $22(\mathrm{~s})$ & $29(\mathrm{~s})$ & Escherichia coli \\
\hline SFL $_{1}$ & $22(\mathrm{~s})$ & $20(\mathrm{~s})$ & $22(\mathrm{~s})$ & $0(\mathrm{r})$ & $0(\mathrm{r})$ & $0(\mathrm{r})$ & $0(\mathrm{r})$ & $0(\mathrm{r})$ & Staphylococcus aureus \\
\hline SAR $_{2}$ & $31(\mathrm{~s})$ & $0(\mathrm{r})$ & $20(s)$ & $12(\mathrm{r})$ & $12(\mathrm{r})$ & $12(\mathrm{r})$ & $26(s)$ & $27(\mathrm{~s})$ & Pseudomonas aeruginosa \\
\hline $\mathrm{SAR}_{3}$ & $0(\mathrm{r})$ & $0(\mathrm{r})$ & $18(\mathrm{~s})$ & $25(\mathrm{r})$ & $0(\mathrm{r})$ & $0(\mathrm{r})$ & $0(\mathrm{r})$ & $25(\mathrm{~s})$ & Staphylococcus aureus \\
\hline $\mathbf{S A R}_{2}$ & $0(\mathrm{r})$ & $16(\mathrm{i})$ & $0(\mathrm{r})$ & $12(\mathrm{r})$ & $0(\mathrm{r})$ & $0(\mathrm{r})$ & $22(\mathrm{~s})$ & $29(\mathrm{~s})$ & Escherichia coli \\
\hline $\mathrm{SAR}_{3}$ & $25(\mathrm{~s})$ & $0(\mathrm{r})$ & $0(\mathrm{r})$ & $25(\mathrm{~s})$ & $0(\mathrm{r})$ & $30(\mathrm{~s})$ & $20(\mathrm{~s})$ & 16(i) & Escherichia coli \\
\hline SDK $_{2}$ & $22(\mathrm{~s})$ & $20(s)$ & $22(s)$ & $0(\mathrm{r})$ & $0(\mathrm{r})$ & $0(\mathrm{r})$ & $0(\mathrm{r})$ & $0(\mathrm{r})$ & Staphylococcus aureus \\
\hline
\end{tabular}

Key: S- Saint Theresa Hospital, AR- Hospital air, BR- Bed Rails, DK- Door knobs, NT- Nurse Table Top, ST-Stretchers, SK-Sink, TS - Toilet seat, OT-

Operation table, FL- Floor, TS - Toilet Seat, $\mathrm{r}=$ resistant, $\mathrm{s}=$ sensitive 
Klebsiella spp were susceptible to ofloxacin, ceftriazone, gentamicin, cefuroxime, augmentin and ceftazidime but resistant to cloxacillin and erythromycin (Table 5). This result corroborates with findings of other scholars that hospitals provide a reservoir of microorganisms, many of which are multi-resistant to antibiotics (Fithamlak et al., 2017).

Meanwhile, E. coli isolated from Federal Medical Centre was sensitive to considerable number of the antibiotics with sensitivity to ofloxacin and augmentin streaking (Table 5). Whereas this was in contrast to the strain of $E$. coli isolates from Saint Theresa Hospital (Table 6). This corroborates with the reports of Gelaw et al. (2013) and Dessie et al. (2016) that stated $E$. coli among gram-negative isolates found resistant to antimicrobial tested.

The highest incidence of Staphylococcus aureus in the work give an ample opportunity of looking into antibiogram of the isolates. It was observed that Staphylococcus aureus isolated from City Hospital were highly sensitive to ofloxacin (inhibitors of nucleic acid synthesis) and bacteriological assessment of Staphylococcus aureus isolated from Saint Theresa Hospital reveals that the bacteria is sensitive to cloxacillin (cell wall inhibitor antimicrobial agent). Staphylococcus aureus isolated from Federal Medical Centre Wadata was also sensitive to ofloxacin (a member of fluoroquinolones) which is capable of inhibiting nucleic acid synthesis. This is in agreement with the report of Pollack (2010), that the effectiveness of antimicrobial drugs in chemotherapy is based on the susceptibility of the target microbes.

In general, predisposing factors that make patients susceptible to nosocomial infections had been reported by some researchers, the length of hospital stay remains the most important factors of nosocomial infections (Kleven et al., 2007; Lahsaeizadeh et al., 2009). Assessment of environmental quality of hospital wards should be based on comprehensive analysis with multiple indicators, because there may be imbalances in the microbial diversity in the hospital wards, therefore, monitoring of the environmental quality of hospitals is important in the prevention of nosocomial infections (Hart et al., 2015; Ling and Hui, 2019; Loupa, et al., 2016). This research is in line with the study on nosocomial bacterial infections and their antimicrobial susceptibility patterns among patients in Ugandan intensive care units (Peter et al., 2017). It is also consistent with the study on evaluation of the complex indoor environment of hospital wards through the composition of bacteria and microbial ecology of the hospital wards (Ling and Hui, 2019).

\section{CONCLUSION}

This investigation has unveiled the hide out of pathogenic bacteria in hospital premises from hospital floor even to the air. Incidence of Gram-negative bacteria is less than incidence of Gram bacteria which mean, patients are prone to contract bacterial infection associated with Gram positive bacteria than Gram negative. This study reveals Klebsiella spp as the predominant bacteria in hospital air. It further reveals $E$. coli, Pseudomonas aeruginosa, S. aureus and other Staphylococcus spp as bacteria commonly associated with hospital environment and these bacteria are capable of causing nosocomial infections on patients with compromised immunity. Antibiotics susceptibility pattern of the bacterial isolates reveals the presence of highly resistant species of $P$. aeruginosa and Klebsiella in the hospitals which has high public health implication. This report will create awareness and be a good guide to health care workers, patients and the public about the likeliness of contracting nosocomial infection and how to treat such infection.

\section{RECOMMENDATIONS}

Based on the findings and conclusion reached above, the following recommendations are hereby made:

1. Healthcare workers should be more careful in carrying out their duty to avoid been infected in the course of work.

2. The in-vitro antibiotics susceptibility testing on the bacterial pathogens in this study shows that erythromycin, Ceftazidime, Cefuroxime and Cloxacillin may not be effective in the treatment of the hospital acquired infections caused by bacterial pathogens. Therefore, information on resistance patterns of isolates encountered in this study will assist the clinicians in making improvement in management of nosocomial infections.

3. Health care workers, patients and any other individual who visit hospitals should keep proper hygiene through hand wash and the use of hand sanitizer.

4. Use of antiseptics to clean inside hospital environments surfaces more than once in a day and use of recommended air fresheners in hospital wards will ameliorate the high loads of the pathogens and reduces the chances of infection by immuno-compromised individuals.

\section{FUTURE WORK}

There should be sound epidemiology and health information system, properly documented for surveillance in hospitals to be able to monitor, control in order to tackle the rate of hospital acquired infections.

\section{ACKNOWLEDGEMENT}

The authors hereby thank the entire staff of the City Hospital Makurdi, Saint Theresa Hospital, and Federal Medical Centre Wadata, Makurdi, Benue State, Nigeria for their technical support received during the research work. 


\section{REFERENCES}

Anusree, V., Neetha, J., Kiran, K., Neenu, T., Kamlesh, J. and Shanta, N. (2015). Environmental Microbiology. A Comparative Study of Coastal and Clinical Isolates of Pseudomonas aeruginosa. Brazilian Journal of Microbiology, 46(3), 725. http://doi.org/10.1590/S1517-838246320140 502 PMid:26413053 PMCid:PMC4568853

Arzu, K., Samyukta, T., Shyaron, P., Sony, S. and Pramila, P. (2019). Bacteriological Assessment of the Indoor Air of Different Hospitals of Kathmandu District. International Journal of Microbiology, Article ID 5320807 https://doi.org/10.1155/2019/5320807 PMid:31089326 PMCid:PMC6476020

Awosika A., Olajubu, A. and Amusa, A., (2012). Microbiological assessment of indoor air of a teaching hospital in Nigeria. Asian Pacific Journal of Tropical Biomedicine, 2(6), 465-468. https://doi.org/10.1016/S2221-1691(12)60077-X

Bereket, W., Hemalatha, K., Getenet, B., Wondwossen, T., Solomon, A., Zeynudin, A. and Kannan, S. (2012). Update on bacterial nosocomial infections. European Review for Medical and Pharmacological Sciences, 16, 1039-1044. Available at: https://www.europeanreview.org/article/1249

Bergey, D. H. and Holt, J. G. (2000). Bergey's manual of determinative bacteriology (9th ed.). Philadelphia: Lippincott Williams and Wilkins.

Brooks, G. F., Carroll, K. C., Butel, J. S., Morse, S. A. and Meitzner T. A. (2010). Jawetz, Melnick and Adelberg's Medical Microbiology (25th ed., pp. 213-219). McGraw-Hill Companies Inc.

Cheesbrough, M. (2006). District Laboratory Practice Manual in Tropical Countries Part 2 (pp. 157-180). Cambridge University Press. https://doi.org/10.1017/ CBO9780511543470 PMCid:PMC2870630

Derese, H., Mekonnen, D., Awoke, D., Wondemagegn, M. and Bayeh, A. (2016). Pathogenic bacteria profile and antimicrobial susceptibility patterns of ear infection at Bahir Dar Regional Health Research Laboratory Center, Ethiopia. Springer Plus, 5, 466. https://doi.org/10.1186/ s40064-016-2123-7 PMID:27119070 PMCID: PMC4833760

Dessie, W., Mulugeta, G., Fentaw, S., Mihret, A., Hassen, M. and Abebe, E. (2016). Pattern of bacterial pathogens and their susceptibility isolated from surgical site infections at selected referral hospitals, Addis Ababa, Ethiopia. International Journal of Microbiology, 2016, 2418902. https://doi.org/10.1155/2016/2418902ＰMid:27446213 PMCid:PMC4944047

Endalafer, N., Gebre-Selassie, S. and Kotisso, B. (2010). Nosocomial bacterial infections in a tertiary hospital in Ethiopia. Journal of Infection Prevention, 12(1), 38-43. https://doi.org/10.1177/1757177410376680

Fithamlak, B., Solomon, F. W. W., Amsalu, A. A. and Yishak, L. A. (2017) Antibiotic resistant airborne bacteria and their multidrug resistance pattern at University teaching referral Hospital in South Ethiopia. Ann Clin Microbiol Antimicrob, 16, 29. https://doi.org/10.1186/s12941-017-0204-2 PMid:28403894 PMCid:PMC5389437
Fu, W. and Wang, S. (2016). Nosocomial infection control in healthcare settings: Protection against emerging infectious diseases. Infectious Diseases of Poverty, 5, 30. https://doi.org/10.1186/s40249-016-0118-9 PMid:27068809 PMCid:PMC4828876

Gelaw, A., Gebre, S. S., Tiruneh, M. and Fentie, M. (2013). Antimicrobial susceptibility patterns of bacterial isolates from patients with post-operative surgical site infection, health professionals and Environmental samples at a tertiary level hospital. International Journal of Pharmaceutical Sciences and Research, 3(1), 1-9.

Hart, J. E., Liao, X., Hong, B., Puett, R. C., Yanosky, J. D., Suh, H., Kioumourtzoglou, M. A., Spiegelman, D. and Laden, F. (2015) The association of long-term exposure to PM2.5 on all-cause mortality in the Nurses' health study and the impact of measurement-error correction. Environ Health., 14, 38. https://doi.org/10.1186/s12940-015-0027-6 PMid:25926123 PMCid:PMC4427963

Hassan, A. K., Aftab, A. and Riffat, M. (2015). Nosocomial infections and their control strategies. Asian Pacific Journal of Tropical Biomedicine, 5(7), 505-509. https://doi.org/10.1016/j.apjtb.2015.05.001

Hayleeyesus, S. F. and Manaye, A. M. (2014). Microbiological quality of indoor air in university libraries. Asian Pacific Journal of Tropical Biomedicine, 4(1) 312-317. https://doi.org/10.12980/APJTB.4.2014C807 PMid:25183103 PMCid:PMC4025286

Kasper, D. L., Braunwald, E., Fauce, A. S., Hauser, S. Longo, D. L. and Jameson, L. J. (2005). Harrison's Manual of Medicine (16th ed.). McGraw Medical publishing division, New York.

Klevens, R. M., Edwards, J. R., Chesley, L. R., Jr., Teresa, C. H., Robert, P. G., Daniel, A. P. and Denise, M. C. (2007). Estimating health care-associated infections and deaths in U.S. hospitals. Public Health Reports, 122(2), 160-166. https://doi.org/10.1177/003335490712200205 PMID:17357358 PMCID:PMC1820440

Lahsaeizadeh, S., Jafari, H. and Askarian, M. (2009). Health care associated infection in Shiraz, Iran 2004-2005. Journal of Hospital Infection, 69, 283-287. https://doi.org/10.1016/j.jhin.2008.05.006 PMID:18550217

Ling, S. and Hui, L. (2019). Evaluation of the complexity of indoor air in hospital wards based on PM2.5, real-time PCR, adenosine triphosphate bioluminescence assay, microbial culture and mass spectrometry. BMC Infect Dis., 19, 646. https://doi.org/10.1186/s12879-019-4249-z PMid:31324234 PMCid:PMC6642494

Loupa, G., Zarogianni, A. M., Karali, D., Kosmadakis, I. and Rapsomanikis, S. (2016). Indoor/outdoor PM2.5 elemental composition and organic fraction medications, in a Greek hospital. Sci Total Environ., 550, 727-735. https://doi.org/10.1016/j.scitotenv.2016.01.070 PMid:26849336

Michels, H. T. (2006). Anti-Microbial Characteristics of Copper, ASTM Standardization News, 28-31. Available at: https://www.astm.org/SNEWS/OCTOBER_2006/michels_o ct06.html 
Nester, E. W., Anderson, D. G., Evans, C. R. and Nester, M. T. (2009). Microbial Ecology. Microbiology: A Human Perspective (6th ed., pp. 721-736), McGraw-Hill Higher Education.

Pallant, J. (2007). SPSS survival manual: a step by step guide to data analysis using SPSS for Windows. Open University Press.

Peter, A., Janat, T., Tindimwebwa, J. V. B. and Arthur, K. (2017). Nosocomial bacterial infections and their antimicrobial susceptibility patterns among patients in Ugandan intensive care units: a cross sectional study. $B M C$ Res Notes, 10, 349. https://doi.org/10.1186/s13104-0172695-5 PMid:28754148 PMCid:PMC5534037

Pollack, A. (2010). Rising Threat of Infections Unfazed by Antibiotics. New York Times.

Prescott, L. M., Harley, J. P. and Klein, D. A. (2008) Biogeochemical Cycling and Introductory Microbial Ecology: General Microbiology (7th ed., pp. 643-665). McGraw-Hill Companies Inc. New York.

Stamm, W. E (2001). Urinary tract infections and pyelonephritis. In: T. R. Harrison and E. Braunwald (eds.), Harrison's Principles of internal medicine (15th ed., pp. 1620 - 1626). McGraw-Hill Companies Inc. New York.
Tambekar, D. H., Gulhane, P. B. and Bhokare, D. D. (2007). Studies on Environmental Monitoring of Microbial Air Flora in the Hospitals. Journal of Medical Sciences, 7, 67-73. https://doi.org/10.3923/jms.2007.67.73

Whitman, W. B., Goodfellow, M., Kämpfer, P., Busse, H.-J., Trujillo, M. E., Ludwig, W. and Suzuki, K. I. (eds.). (2012). Bergey's Manual of Systematic Bacteriology (2nd ed., vol. 5, parts A and B). New York, NY: Springer-Verlag. https://doi.org/10.1007/978-0-387-68233-4

WHO (2002). Prevention of hospital acquired infections: A practical guide. Malta: Department of Communicable Disease, Surveillance and Response. Available at: http://apps.who.int./iris/bitstream/handle/10665/67350/ WHO_CDS_CSR_EPH_2002.12.pdf (Accessed: 31 May 2020).

Wilks, S. A., Michels, H. and Keevil, C. W. (2005). The Survival of Escherichia coli 0157 on a Range of Metal Surfaces. International Journal of Food Microbiology, 105(3), 445-454. https://doi.org/10.1016/j.ijfoodmicro.2005.04.021 PMid:16253366

Wondemagegn, M., Gebre, K., Getenet, B. and Meku, D. (2012). Postoperative Nosocomial Infections and Antimicrobial Resistance Pattern of Bacteria Isolates among Patients Admitted at FelegeHiwot Referral Hospital, Bahirdar, Ethiopia. Ethiopian Journal of Health Science, 22(1), 7-18. PMCID:3437975 PMID:22984327 